\title{
Fano Factor of Chromosome Aberrations and Asessment of Repair Efficiency
}

\author{
A. Kowalska ${ }^{a, *}$, W. Pereira ${ }^{b}$ And K. Czerski ${ }^{b}$ \\ ${ }^{a}$ Maritime University of Szczecin, Faculty of Marine Engineering, Szczecin, Poland \\ ${ }^{b}$ University of Szczecin, Faculty of Physics, Szczecin, Poland
}

\begin{abstract}
A new statistical method to study repair mechanism of individual cells exposed to ionizing radiation is presented. The method is based on deviations from the Poisson distribution of observed chromosome aberrations and allows to determine the efficiency of repair mechanisms of individual cells. On basis of the dose-effect curves and the chi-square analysis, the ratio between the variance and the mean value of the observed aberration distribution could be calculated and related to the efficiency of the cellular repair mechanisms. The method has been tested on human lymphocytes and CHO-K1 cells exposed to different radiation species. In the case of high-linear energy transfer ion irradiation a correction due to Neyman A statistics has been introduced.
\end{abstract}

DOI: 10.12693/APhysPolA.133.225

PACS/topics: 87.10.Mn, 87.15.ht, 87.53.Ay, 87.15.ad

\section{Introduction}

Chromosome aberrations (CA) induced by ionizing radiation comprise very sensitive biomarker of genetic damage. Aberrations arise from non-repaired or mis-repaired DNA double strand breaks (DSB) and can be directly observed under optical microscope after using standard staining techniques. Differences in the microscopic dose deposition for radiation quality applied (due to different linear energy transfer, LET) lead to different distributions of CA among cells which is, in turn, of special interest of the hadron radiotherapy. CA induced by low-LET radiation species are randomly distributed over a cell nucleus, therefore can be described by Poisson statistics [1]. On the other hand, high-LET swift charged particles create in the irradiated tissue well localized ion tracks and the CA probability results from convolution of two independent Poisson statistics. First gives the probability that cell nucleus will be hit while the second determines the probability of aberration induction by each hit. In that case, we speak about Neyman A statistics [2].

Repair mechanisms of individual cells are very effective and can be well assessed for example by means of the comet assay method [3] or by the $\gamma$-H2AX foci monitoring [4]. The question is, however, whether the cell repair efficiency can be also estimated by applying statistical methods - especially regarding $\mathrm{CA}$ which, in comparison to the comet assay and foci, are sensitive to details of the rejoining processes. From the statistical point of view, repair may lead to the reduction of the distribution variance of aberration frequencies. Such an effect has been already observed for experimentally determined CA distributions and described statistically by the generalized Poisson distribution (GPD) [5]. Nevertheless, GPD

*corresponding author; e-mail: a.kowalska@am.szczecin.pl cannot be applied unambiguously for low doses, since the fitting procedure needs large number of seldom multiple aberrations.

We have proposed a new statistical method to study the efficiency of repair mechanisms $[6,7]$ which is based on the estimation of the deviation from the Poisson distribution observed for the dose-effect curves and applicable for relatively low doses at which multiple aberrations are difficult to observe. According to the proposed method, the ratio between the variance and the mean value of the observed aberration distribution is directly related to the efficiency of the cellular repair mechanisms. The latter can be interpreted as the Fano factor which was originally introduced to describe reduction of the noise in the charge particle detectors [8]. In the present work, we expand the method for high-LET radiations for which the inhomogeneous dose deposition follows Neyman A statistics.

\section{Materials and methods}

Proposed method was applied to experimental data obtained for human peripheral blood lymphocytes (PBL) exposed to different doses of ${ }^{60} \mathrm{Co} \gamma$ irradiation, $150 \mathrm{MeV}$ protons, $199 \mathrm{MeV} / \mathrm{u}$ carbon ions, and $22 \mathrm{MeV} / \mathrm{n}$ boron ions, presented as dose-effect curves in Fig. 1. Donors were healthy volunteers of both sex. Each dose-effect curve was separately measured for one person only to reduce systematical uncertainties. The number of cells scored amounted to 100-200 for each data point.

To demonstrate universality of observed effects, we also analyze the data obtained by other authors [9] for Chinese hamster ovary fibroblasts (CHO-K1) irradiated by ${ }^{60} \mathrm{Co} \gamma$ and $0.8 \mathrm{MeV} / \mathrm{n}$ carbon ions.

In the previous experiments, it has been recognized that error bars calculated according to the Poisson statistics (see Fig. 1) are overestimated in comparison to the dispersion of data points around the fitted dose-response 
curve [6]. In the case of the Poisson statistics, about one third of the points should be away from the fitted (according to the linear-quadratic LQ model) curve.
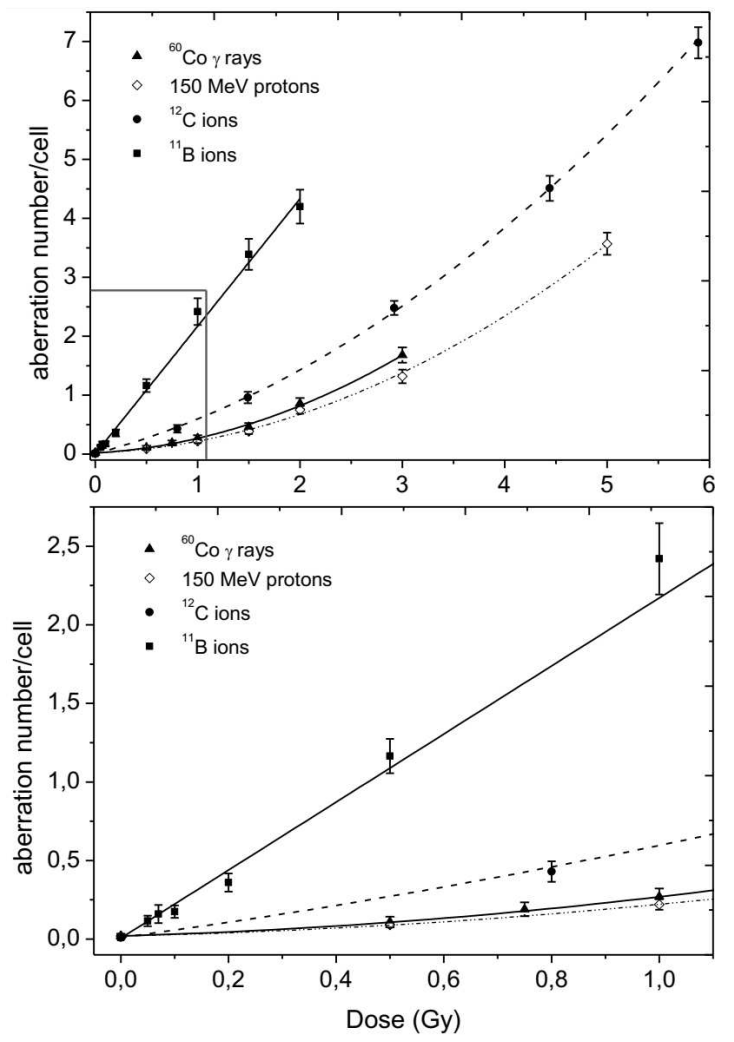

Fig. 1. Dose response curves of chromosome aberrations observed in human lymphocytes. Aberration number per individual cell $(\mathrm{Y})$ has been fitted according to the LQ model: $Y=\alpha D+\beta D^{2}+i n t$ for CA induced by $150 \mathrm{MeV}$ protons, carbon ions and $\gamma$ rays and $Y=\alpha D+i n t$ for CA induced by boron ions [10]. Upper part represents the whole investigated dose spectra. Low part represents a piece of the data at the dose range below 1 Gy.

As a measure of statistical variance, the distances of the experimental points to the theoretical curve can be calculated using the expression for the $\chi^{2}$ function, where $\sigma_{i}$ stands for the experimental standard deviation. For the linear-quadratic fit, $\chi^{2}$ function reads as follows:

$$
\chi^{2}=\frac{1}{n-3} \sum_{i=1}^{n} \frac{\left(Y_{i}-i n t-\alpha D_{i}-\beta D_{i}^{2}\right)^{2}}{\sigma_{i}^{2}},
$$

where int denotes the interception of fitted curve. For linear fit

$$
\chi^{2}=\frac{1}{n-2} \sum_{i=1}^{n} \frac{\left(Y_{i}-i n t-\alpha D_{i}\right)^{2}}{\sigma_{i}^{2}} .
$$

If the distances of the experimental points to the fitted line would correspond to the really observed standard deviation $\sigma_{R i}$, the $\chi^{2}$ value should be equal to one. Assuming that real standard deviations $\sigma_{R i}$ are smaller by a factor $k$ than those calculated according to Poisson statistics $\sigma_{P_{i}}$ the following relation can be obtained:

$$
\begin{gathered}
\chi_{P}^{2}=\frac{1}{n-3} \sum_{i=1}^{n} \frac{\left(Y_{i}-i n t-\alpha D_{i}-\beta D_{i}^{2}\right)^{2}}{\sigma_{P i}^{2}}= \\
\frac{1}{k^{2}} \frac{1}{n-3} \sum_{i=1}^{n} \frac{\left(Y_{i}-i n t-\alpha D_{i}-\beta D_{i}^{2}\right)^{2}}{\sigma_{R i}^{2}}= \\
\frac{1}{k^{2}} \chi_{R}^{2}=\frac{1}{k^{2}} .
\end{gathered}
$$

Overestimation of the variance may be explained by the occurrence of highly efficient repair mechanisms $[6,7]$ which reduces originally produced damage yield $Y^{\prime}$ which could be observed in the lack of any repair. Finally detected aberration yield $Y$ is the result of a decrease of the number of originally induced DNA double strand breaks due to the DNA repair processes. As a measure of repair efficiency, the repair factor $R F$ has been defined:

$$
R F=\frac{Y^{\prime}}{Y} \geqslant 1 \text {. }
$$

Assuming that the aberration yields may be also expressed by the corresponding variances and the factor $k$ is constant $(k \leqslant 1)$, the repair factor may be calculated as follows:

$$
R F=\frac{\sigma_{P}^{2}}{\sigma_{R}^{2}}=\frac{\chi_{P}^{2}}{\chi_{R}^{2}}=\chi_{P}^{2}=\frac{1}{k^{2}} .
$$

For a high-LET exposure $\left({ }^{11} \mathrm{~B}\right.$ irradiation) following the Neyman A statistics, the variance is given by: $\sigma_{N}^{2}=$ $\lambda_{P}(1+\mu)$, where $\lambda_{P}$ represents mean number of aberrations per cell nucleus and $\mu$ is the mean number of aberrations induced by each hit. Thus, for small $\mu$ values we get the Poisson variance.

According to the proposed method we can also define repair coefficients $R C$, which represents the percentage of repaired chromosomes. The expression for $R C$ reads as follows:

$$
R C=\frac{R F-1}{R F} \times 100 \% .
$$

\section{Results}

For each set of the experimental data $\chi^{2}$ values, repair factors and repair coefficients have been presented (see Table I).

Comparison of $\mathrm{RF}$ and $\mathrm{RC}$ values

TABLE I obtained for PBL and CHOK1 cells.

\begin{tabular}{c|c|c|c|c|c}
\hline \hline $\begin{array}{c}\text { Cell } \\
\text { type }\end{array}$ & $\begin{array}{c}\text { Radiation } \\
\text { species }\end{array}$ & $\begin{array}{c}\text { LET } \\
{[\mathrm{keV} / \mu \mathrm{m}]}\end{array}$ & $\chi^{2}$ & $R F$ & $\begin{array}{c}R C \\
{[\%]}\end{array}$ \\
\hline \multirow{5}{*}{$\mathrm{PBL}$} & ${ }^{60} \mathrm{Co} \gamma$ rays & 0.2 & $0.31 \pm 0.20$ & $3.2 \pm 2.0$ & $69 \pm 20$ \\
& $150 \mathrm{MeV}$ p & 0.57 & $0.37 \pm 0.23$ & $2.7 \pm 1.7$ & $63 \pm 23$ \\
& $22 \mathrm{MeV} / \mathrm{n}^{12} \mathrm{C}$ & 16 & $0.26 \pm 0.18$ & $3.8 \pm 2.7$ & $73 \pm 18$ \\
& $199 \mathrm{MeV} / \mathrm{n}^{11} \mathrm{~B}$ & 76 & $0.43 \pm 0.21$ & $2.3 \pm 1.1$ & $57 \pm 21$ \\
\hline \multirow{2}{*}{$\mathrm{CHOK} 1$} & ${ }^{60} \mathrm{Co} \gamma$ rays & 0.2 & $0.006 \pm 0.0025$ & $160 \pm 70$ & $99.0 \pm 0.3$ \\
& $8.09 \mathrm{MeV}^{12} \mathrm{C}$ & 830 & $0.44 \pm 0.28$ & $2.3 \pm 1.4$ & $56 \pm 28$
\end{tabular}

\section{Discussion and conclusions}

For all applied irradiations, we observe that the variance of experimental data points measured as $\chi^{2}$ values obtained for the dose-effect curves are much smaller than 
expected for the Poisson statistics. The measure for this reduction is the repair factor which is larger than unity whereas the repair coefficient describes how efficient are the cellular repair mechanisms (see Table I). We would expect that radiations of larger LET and higher ionization density induce damages which are much more difficult to repair. This trend can be clearly confirmed for the data presented in Table I. Only the repair coefficient for ${ }^{12} \mathrm{C}$ is relatively large but the corresponding uncertainty is also high. A similar tendency illustrating generality of the method can be observed for experimental results obtained on CHOK1 cells for which efficiency of repair is different. On the other hand, the strength of the repair mechanisms can be determined by means of curvature of the dose-effect curves, as well. In that case, we assumed that RC depends on the dose. The dependence is, however, very weak and therefore is not in contradiction to the present results.

\section{References}

[1] A.A. Edwards, D.C. Lloyd, R.J. Purrott, Radiat. Env. Biophys. 16, 89 (1979).

[2] R. Virsik, D. Harder, Radiat. Res. 85, 13 (1981).

[3] P.L. Olive, Mutat. Res. 681, 13 (2009).

[4] Li-Jeen Mah, C. Orlowski, K. Ververis, R.S. Vasireddy, Assam El-Osta, T.C. Karagiannis, Genome Integr. 2, 1 (2011).

[5] C.M.S. Lee, F.A. Famoye, Biom. J. 38, 299 (1996).

[6] A. Kowalska, K. Czerski, M. Kaczmarski, M. Lewocki, B. Masojć, A. Łukowiak, Eur. Phys. J. D 69, 3 (2015).

[7] A. Kowalska, K. Czerski, M. Kaczmarski, W. Pereira, P. Kutsalo, E. Zaytseva, Acta Bio-Optica Inf. Medica 21, 24 (2015).

[8] U. Fano, Phys. Rev. 72, 26 (1947).

[9] J. Czub, D. Banaś, A. Błaszczyk, J. Braziewicz, I. Buraczewska, J. Choiński, U. Górak, M. Jaskóła, A. Korman, A. Lankoff, H. Lisowska, A. Łukaszek, Z. Szefliński, A. Wójcik, Appl. Radiat. Isot. 67, 447 (2009).

[10] A. Kowalska, E. Nasonova, K. Czerski, P. Kutsalo, E. Krasavin, Eur. Phys. J. D 71, 332 (2017). 